\title{
High Speed Pilot-less Sampling Frequency Acquisition for DMT Systems
}

\author{
Ching-Chi Chang and Chorng-Kuang Wang \\ Graduate Institute of Electronics Engineering and Department of Electrical Engineering \\ National Taiwan University \\ Taipei 106, Taiwan R.O.C. \\ ckwang@cc.ee.ntu.edu.tw
}

\begin{abstract}
This paper presents a sampling frequency offset estimation in time domain for discrete multitone (DMT) systems. Cyclic prefix (CP) enables this estimation without pilots. The proposed algorithm realizes sampling frequency estimation on each sample within the last period where the $C P$ copied from. The sampling-rate iteration method markedly improves acquisition speed and jitter performances. Simulations show that this method can synchronize DMT systems in both AWGN channels and twisted pair copper wires along with non-ideal factors. Practical examples of the veryhigh bit-rate digital subscriber lines (VDSL) are used to illustrate results.
\end{abstract}

\section{INTRODUCTION}

Discrete multitone (DMT) modulation is recognized to efficiently transmit data over spectrally shaped noisy channels [1], and has been standardized for broadband communications over existing copper networks $[2,3]$. The significant drawback of DMT systems is the sensitivity to synchronization errors, including DMT symbol offset and mismatched sampling clocks. The receiver has to employ a timing recovery to correct the sampling clock since intercarrier-interference (ICI) power is proportional to the square of the sub-carrier index and the sampling clock offset [4].

Practically, an adaptive receiver is preferable to combat varying transmission environments. The timing recovery can utilize a digital phase-locked loop (PLL) to acquire and maintain the sampling clock, thus, the bandwidth and the sampling rate of the PLL dominate the acquisition speed [5]. The state-of-the-art timing estimations depend on observing rotational pilots in the frequency domain $[4,8]$. Since pilots arrive at the symbol rate, e.g. about $4 \mathrm{kHz}$ in VDSL systems, the PLL must be narrow bandwidth and take a long time to compensate the sampling clock, e.g. 200-symbol time in VDSL systems $[6,7]$. As the sub-carrier number grows, the propagation error of the sampling phase degrades the jitter performance of the timing recovery. Besides, if the occasional radio frequency interference (RFi) exists in the corresponding frequency of the pilot, the synchronization mechanism collapses.

In this paper, we present a time domain estimation method which can directly calculate each phase error between the samples in the cyclic prefix $(\mathrm{CP})$ and its repeat within a DMT symbol. By applying a proportional-andintegral (PI) filter in the PLL, the receiver can estimate and compensate the sampling clock at the analog-to-digital (A/D) sampling rate, e.g. $35 \mathrm{MHz}$ in VDSL systems.

This paper organizes as follows. Section II introduces the DMT system model. Section III presents the synchronization in DMT systems. Section IV derives the proposed time domain timing estimation method for DMT systems. Simulations and practical considerations are shown in Section V. And the conclusions are drawn in Section VI.

\section{DMT SYSTEM MODEL}

The DMT transceiver, shown in Fig. 1, splits the available bandwidth into a number of sub-bands. In each sub-band a QAM-modulated sub-carrier is transmitted. Denote $X_{n, k}$ the constellation point which modulates the $k$ th sub-carrier during the $n$th DMT symbol. An $N$-point Inverse Fast Fourier Transform (IFFT) digitally performs modulations. In order to generate real signals, $X_{n, k}$ must satisfy $X_{n, 0}=0$ and $\left(X_{n, k}\right)^{*}=X_{n, N-k}, k \in[0, N-1]$. An appended $L$ sample CP guarantees orthogonal signals at the receiver. The digital-to-analog $(\mathrm{D} / \mathrm{A})$ converter which runs at the rate $1 / T$ transmits the continuous signal $s(t)$,

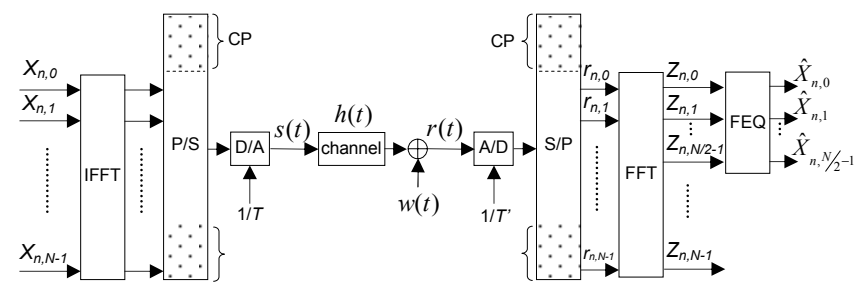

Fig. 1. The DMT transceiver system. 


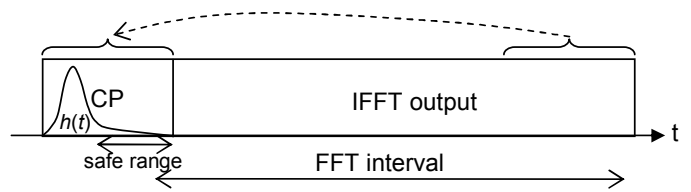

Fig. 2. A typical DMT symbol.

$$
s(t)=\frac{1}{N} \sum_{n=-\infty}^{\infty} \sum_{k=0}^{N-1} X_{n, k} \psi_{n, k}(t)
$$

where

$$
\begin{aligned}
& \psi_{n, k}(t)=e^{j 2 \pi f_{k}\left(t-T_{g}-n T_{s}\right)} u\left(t-n T_{s}\right), \\
& u(t)= \begin{cases}1, & 0 \leq t<T_{s} \\
0, & \text { else. }\end{cases}
\end{aligned}
$$

$T_{u}$ and $T_{g}$ are IFFT and CP intervals respectively, and $T_{s}=T_{u}+T_{g}$. The sampling period $T=T_{u} / N$, and the sub-carrier frequency $f_{k}=k / T_{u}, k \in[0, N-1]$.

In the receiver, the $\mathrm{A} / \mathrm{D}$ converter digitizes the received signal $r(t)$ at the rate $1 / T^{\prime}$, where

$$
r(t)=s(t) \otimes h(t)+w(t),
$$

$h(t)$ is the impulse response of the channel, and $w(t)$ is the AWGN. In order to detect the transmitted symbols, the receiver processes blocks of $N+L$ consecutive samples and removes the first $L$ samples of each block. The FFT demodulates the remaining samples and outputs

$$
Z_{n, m}=X_{n, m} H_{n, m}+W_{n, m}, m=0,1, \ldots, N-1 \text {, }
$$

where $H_{n, m}$ and $W_{n, m}$ are channel and AWGN frequency responses at the $m$ th sub-carrier during the $n$th DMT symbol. A frequency domain equalizer (FEQ) multiplies each indexed FFT output a complex tap to perform channel equalization.

\section{SYNCHRONIZATION IN DMT SYSTEMS}

In order to find the start point of the FFT interval within the received consecutive samples, the receiver can estimate the correlation of $\mathrm{CP}$ and its repeat, and arrange the FFT interval to locate its start point into the safe range as shown in Fig. 2 [4]. Under AWGN channels, the received signal is

$$
r(t)=s(t-l T)+w(t),
$$

where $l$ contains an integer part which is the residual DMT symbol offset and a fraction part which is the A/D sampling phase offset. Using an unsynchronized A/D sampling period $T^{\prime}=(1+\delta) T$, the samples of the $n$th DMT symbol fed to FFT are

$$
\begin{aligned}
r_{n, q} & =\frac{1}{N} \sum_{k=0}^{N-1} X_{n, k} e^{j 2 \pi f_{k}(q+q \delta-l) T}+w_{n, q} \\
& =\frac{1}{N} \sum_{k=0}^{N-1} X_{n, k} e^{j \frac{2 \pi k}{N}(q+q \delta-l)}+w_{n, q}
\end{aligned}
$$

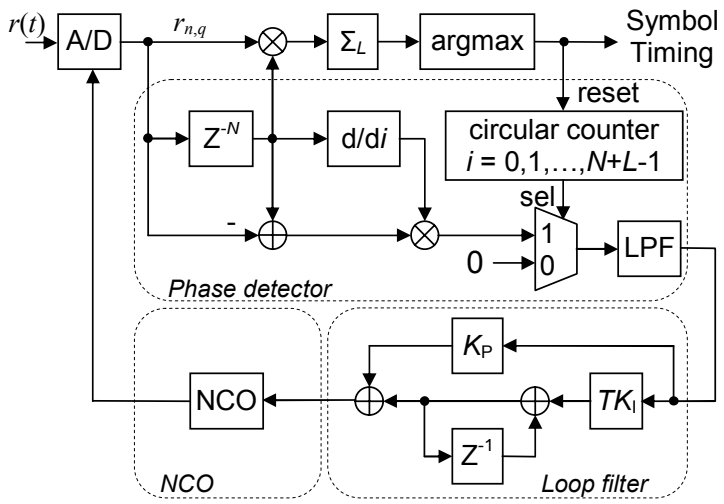

Fig. 3. The proposed timing recovery architecture, it integrates the symbol timing and the sampling frequency synchronizations.

where $q \in[0, N-1]$. After FFT, the demodulated sub-carriers of the $n$th DMT symbol are

$$
\begin{aligned}
Z_{n, m}= & \sum_{q=0}^{N-1} r_{n, q} e^{-j \frac{2 \pi q m}{N}}+W_{n, m} \\
= & \frac{1}{N} \sum_{k=0}^{N-1} X_{n, k} \frac{e^{-j \frac{2 \pi k l}{N}}\left[1-e^{j 2 \pi k(1+\delta)} e^{-j 2 \pi n}\right]}{1-e^{j \frac{2 \pi k}{N}(1+\delta)} e^{-j \frac{2 \pi n}{N}}+W_{n, m}} \\
= & \frac{1}{N} X_{n, m} e^{-j \frac{2 \pi n l}{N}} e^{j \pi n \delta \frac{N-1}{N}} \frac{\sin (\pi m \delta)}{\sin (\pi m \delta / N)}+W_{n, m} \\
& +\frac{1}{N} \sum_{\substack{k=0 \\
k \neq m}}^{N-1} X_{k} e^{-j \frac{2 \pi k l}{N}} e^{j \pi(k+k \delta-m) \frac{N-1}{N}} \frac{\sin (\pi(k+k \delta-m))}{\sin (\pi(k+k \delta-m) / N)},
\end{aligned}
$$

where $m \in[0, N-1]$. If the $\mathrm{A} / \mathrm{D}$ sampling frequency synchronizes with that of the $\mathrm{D} / \mathrm{A}$ in the transmitter, $\delta=0$, and

$$
Z_{n, m}=X_{n, m} e^{-j \frac{2 \pi n l}{N}}+W_{n, m}, \quad m=0,1, \ldots, N-1 .
$$

The influence on each demodulated sub-carrier is the static constellation rotation whose phase is proportional to the subcarrier index $m$. FEQ can easily compensate this phase error.

If A/D sampling frequency drifts, non-zero $\delta$ will alter $l$ and gradually rotate each sub-carrier in consecutive DMT symbols. Also, the magnitude of each sub-carrier varies and ICI appears. The pilot aided estimation algorithms have been well derived $[4,6,7,8]$. Owing to running at symbol rate $1 / T_{s}$, the disadvantage of frequency domain synchronization methods is involved in a tedious acquisition process.

\section{TIME DOMAIN Estimation METHOD}

To speed up the acquisition, the proposed time domain estimation method, shown in (10), evaluates each phase error of the samples in the $\mathrm{CP}$ and its repeat at the sampling rate $1 / T^{\prime}$. The functional block, shown in Fig. 3, employs a digital PLL to track the frequency offset and maintain the jitter 
performance [5]. Following (5), the samples of the $n$th DMT symbol are

$$
r_{n, q}=\frac{1}{N} \sum_{k=0}^{N-1} X_{n, k} e^{j 2 \pi f_{k}(q-L+q \delta-l) T}+w_{n, q},
$$

where $q \in[0, N+L-1]$. The proposed phase error estimation of the phase detector (PD) is

$$
P D=\left\{\begin{array}{l}
r_{n, i-N}^{\prime} \cdot\left(r_{n, i}-r_{n, i-N}\right), i=N, \ldots, N+L-1 \\
0 \quad, \text { else },
\end{array}\right.
$$

where $r_{n, i-N}$ and $r_{n, i}$ are received samples within CP and its repeat of each DMT symbol respectively, and $r_{n, i-N}^{\prime}$ is the differential of $r_{n, i-N}$. Denote $X_{n, k}=X_{n, k}^{R}+j X_{n, k}^{I}$,

$$
\begin{aligned}
& r_{n, i}-r_{n, i-N} \\
& =\frac{-2}{N} \sum_{k=0}^{N-1}\left\{X_{n, k}^{R} \cdot \sin \pi f_{k} N \delta T \cdot \sin 2 \pi f_{k}(i-L-l+(i-N / 2) \delta) T\right. \\
& \left.\quad+X_{n, k}^{I} \cdot \sin \pi f_{k} N \delta T \cdot \cos 2 \pi f_{k}(i-L-l+(i-N / 2) \delta) T\right\} \\
& +w_{n, i}-w_{n, i-N},
\end{aligned}
$$

given $\delta$ approaches to zero, the previous equation approximates

$$
-2 \pi \delta T \sum_{k=0}^{N-1} f_{k}\left(\begin{array}{l}
X_{n, k}^{R} \sin 2 \pi f_{k}(i-L-l) T \\
+X_{n, k}^{I} \cos 2 \pi f_{k}(i-L-l) T
\end{array}\right),
$$

multiplying it with $r_{n, i-N}^{\prime},(10)$ becomes

$$
\begin{aligned}
P D & =\frac{4 \pi^{2} T^{2} \delta}{N}\left\{\sum_{k=0}^{N-1} f_{k}\left(\begin{array}{l}
X_{n, k}^{R} \cdot \sin 2 \pi f_{k}(i-L-l) T \\
+X_{n, k}^{I} \cdot \cos 2 \pi f_{k}(i-L-l) T
\end{array}\right)\right\}^{2} \\
& =\frac{4 \pi^{2} T^{2} \delta}{N}\{D C \text { term }+ \text { AC terms }\},
\end{aligned}
$$

where $i \in[N, N+L-1]$, and $\mathrm{PD}=0$ for else. After the loop filter, PI filter, the control signal fed to NCO is

$$
K_{o} \frac{4 \pi^{2} T^{2} \delta}{N}(D C \text { term })=K_{o} \cdot K_{d} \cdot \delta,
$$

where $i \in[N, N+L-1]$, and $K_{o}$ is the loop filter DC gain. During the start-up protocol, BPSK or QPSK signal is transmitted, the phase detector gain $K_{d}$ is

$$
\frac{4 \pi^{2} T^{2}}{N} \sum_{k=0}^{N-1} f_{k}^{2}
$$

\section{System SimUlation}

In the VDSL system simulations, the DMT symbol consists of 8832 samples, including 8192 modulated Hermitian symmetric sub-carriers and a 640 -sample cyclic extension [3]. The transmission environment comprises of the AWGN of $80 \mathrm{~dB}$ transmitted SNR, twisted pair channel models, far-end cross talks (FEXT), restricted amateur radio bands, and the RFi which is attenuated $30 \mathrm{~dB}$ by the cancellation in the analog front end [3, 7, 9]. The transceiver behaves finite word length and pipeline latencies, and employs both the proposed time domain estimation (TDE) and the frequency domain pilot-aided estimation (FDE) for comparisons. Based on (10) and (15), the TDE uses $\left(r_{n, i-N+1^{-}}\right.$ $r_{n, i-N-1)} / 2 T$ to approximate $r_{n, i-N}^{\prime}$ and a shortened running period, $i \in\left[N+L_{1}, N+L_{2}\right]$, where $L_{2}-L_{1}<L$, to tolerate residual symbol offset and inter-symbol-interference (ISI) caused by channel dispersion.

Fig. 4 shows A/D sampling frequency acquisitions of both estimations under the AWGN channel of $80 \mathrm{~dB}$ SNR. The given $\mathrm{A} / \mathrm{D}$ sampling frequency offsets $100 \mathrm{ppm}$ of the D/A frequency. The TDE takes only 5 DMT symbols while the FDE takes over 150 symbols to reach the requirement of $2 \mathrm{ppm}$ jitter tolerance. Giving the same A/D sampling frequency offset, Fig. 5 shows RMS errors of the compensated $\mathrm{A} / \mathrm{D}$ sampling frequencies in the steady state. Under AWGN channels, the TDE gains $20 \mathrm{~dB}$ SNR performances over the FDE. The advantage of the proposed TDE is to quickly depress the propagating sampling jitter.

Fig. 5 also shows that even the condition is the AWGN along with the twisted pair channel model (1000 ft.), the TDE gains $15 \mathrm{~dB}$ SNR performances over the FDE. ISI caused by the channel dispersion degrades jitter performances. The TDE loses $10 \mathrm{~dB}$ and the FDE loses $5 \mathrm{~dB}$ when the given SNRs are less than $60 \mathrm{~dB}$ and $80 \mathrm{~dB}$ respectively. The disadvantage of the TDE is the sensitivity to ISI. When the given SNRs are higher than the two corner values, the ISI contributes the noise floor and clips the jitter performances of both estimations.

Fig. 6 and 7 show A/D sampling frequency acquisitions of both estimations over $1000 \mathrm{ft}$. and $3000 \mathrm{ft}$. VDSL transmission environments, and Fig. 8 shows A/D sampling frequency RMS errors of both estimations in the steady state under different channels. In the $1000 \mathrm{ft}$. case, the RFi and the FEXT dominate the noise floor for the TDE while only the FEXT influences the FDE due to fine pilot assignment. The proposed TDE possesses high speed acquisition and good jitter performance over the FDE. In the $3000 \mathrm{ft}$. case, the FEXT power decreases, but the ISI and the channel attenuation degrade jitter performances of the TDE.

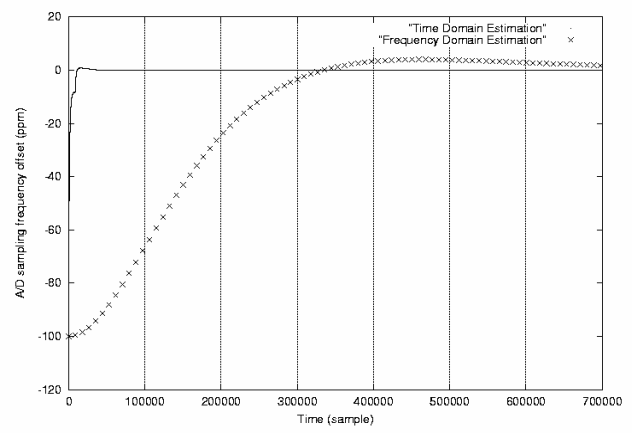

Fig. 4. A/D sampling frequency acquisitions under AWGN channels of 80dB SNR. (Axis X represents the time domain, 1 symbol $=8832$ samples.) 


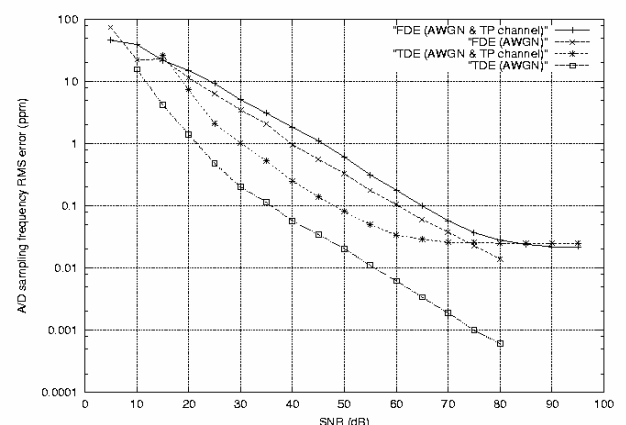

Fig. 5. A/D sampling frequency RMS errors vs. received signal SNRs under either AWGN channels or the AWGN with a $1000 \mathrm{ft}$. twisted pair (TP) channel model.

\section{CONCLUSIONS}

This paper presents a time domain sampling frequency offset estimation method for DMT systems. When this method performs in VDSL systems under practical transmission environments, the timing recovery improves the acquisition speed more than 10 times of the pilot-aided frequency domain estimation method. And the jitter performances lead a gap for $1000 \mathrm{ft}$. transmission range and keep the same level for $3000 \mathrm{ft}$. range. Although this algorithm is sensitive to ISI, the proposed time domain estimation can avoid the failure of the timing recovery when the RFi hits pilots. Since this algorithm takes the advantage of the CP in DMT symbols, the estimation method can be applied to orthogonal frequency division multiplexing (OFDM) and single carrier modulation using frequency domain equalizer (SC-FEQ) systems.

\section{REFERENCES}

[1] J. Bingham, "Multi-carrier modulation for data transmission: an idea whose time has come," IEEE Communications Magazine, vol. 28, no. 5, May 1990, pp. 5-14.

[2] ANSI T1.413, "Network and customer installation interfaces: asymmetric digital subscriber line (ADSL) metallic interface," American National Standards for Telecommunications, June 12, 1998.

[3] Working Group T1E1 (DSL Access), "Very-high bit-rate digital subscriber lines (VDSL) metallic interface," Vancouver, Canada, February 2000

[4] T. Pollet and M. Peeters, "Synchronization with DMT modulation," IEEE Communications Magazine, April 1999, pp. 80-86.

[5] R. E. Best, Phase-Locked Loops, 3rd ed., McGraw-Hill, 1998.

[6] C. C. Chang, M. S. Wang, and T. D. Chiueh, "Design of a DMTbased baseband transceiver for very-high-speed digital subscriber lines, "IEEE Asia-Pacific ASIC Conference, Taipei, Taiwan, Aug. 2002, pp. 367-370.

[7] C. C. Chang, M. T. Shiue, and C. K. Wang, " A VLSI architecture of DMT based transceiver for VDSL system," IEEE Asia-Pacific ASIC Conference, Taipei, Taiwan, Aug. 2002, pp. 363-366.

[8] M. Sliskovic, "Carrier and sampling frequency offset estimation and correction in multicarrier systems," IEEE GLOBECOM, Nov. 2001.

[9] D. J. Rauschmayer, ADSL/VDSL Principles, MTP, 1999.

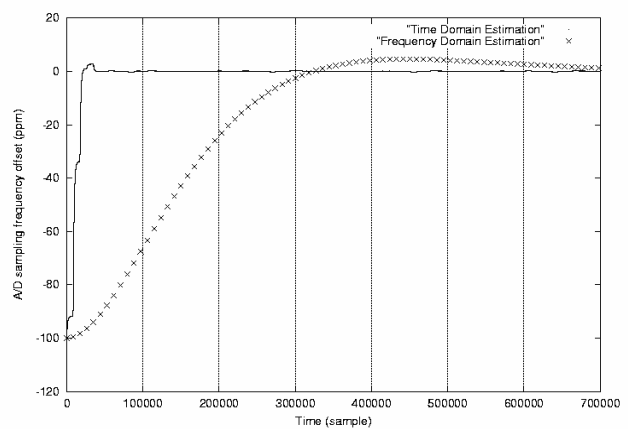

Fig. 6. A/D sampling frequency acquisitions over $1000 \mathrm{ft}$. transmission range.

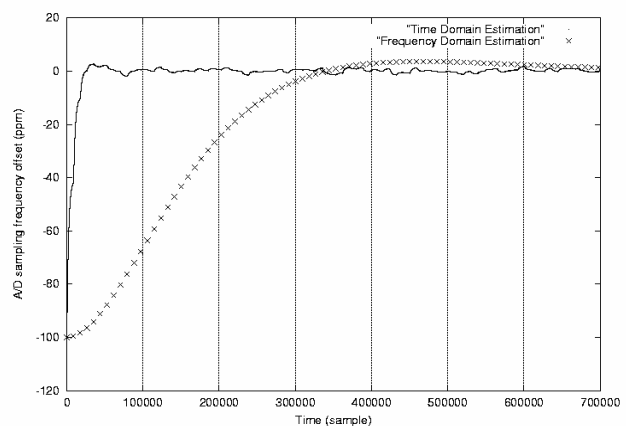

Fig. 7. A/D sampling frequency acquisitions over $3000 \mathrm{ft}$. transmission range.

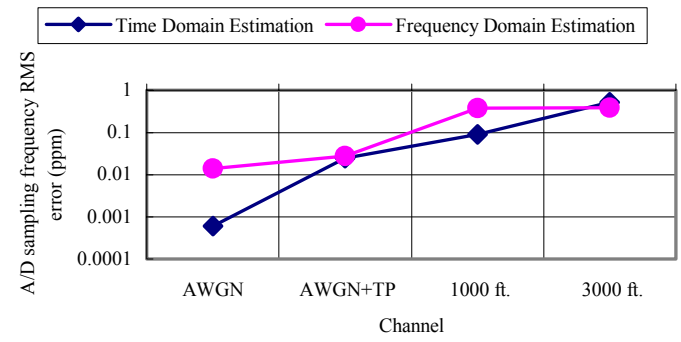

Fig. 8. A/D sampling frequency RMS errors for different channels, including the AWGN of $80 \mathrm{~dB}$ SNR, the AWGN with a $1000 \mathrm{ft}$. TP channel model, $1000 \mathrm{ft}$. transmission range, and $3000 \mathrm{ft}$. transmission range. 